\section{Primäre Kombinationstherapie auch bei Älteren?}

Jüngere Patienten mit einer chronisch obstruktiven

Lungenerkrankung (COPD) haben niedrige ExazerbationsRaten, wenn die Gabe von Beta-Agonisten mit inhalativen Kortikosteroiden (ICS) ergänzt wird. Das Forscherteam um A. S. Gershon aus Toronto / Kanada, ist jetzt der Frage nachgegangen, ob auch Ältere mit Begleiterkrankungen von der Kombination profitieren.

JAMA 2014; 312: 1114-1121

Die Informationen für die retrospektive Kohortenstudie stammten aus Datenbanken des Gesundheitswesens, der Krankenkassen, dem Bevölkerungsregister und der Pharmazie. Außerdem flossen Informationen aus dem landesweiten Canadian Community Health Survey ein. Insgesamt wurden 3258 und 34289 Patienten über 65 Jahre untersucht, die neu auf langwirksame Beta-Agonisten (LABA) bzw. die Kombination aus LABA und ICS eingestellt wurden. 3160 bzw. 8712 Pa- tienten aus den beiden Gruppen wurden für 2,5 bzw. 2,7 Jahre beobachtet.

Der primäre beobachtete Endpunkt aus Mortalität und Krankenhauseinweisungen trat im Kombinationsarm seltener ein (Hazard Ratio [HR] 0,92; 95\%-Konfidenzintervall $[\mathrm{KI}] 0,88-0,96)$. Auch wenn beide Mortalität und Krankenhauseinweisungen getrennt analysiert wurden, hatten mit LABA behandelte Patienten ungünstigere Ergebnisse. Die Fraktur- und Pneu- monieraten unterschieden sich nicht. Unter Berücksichtigung von Rauchen, BodyMass-Index und subjektiver Gesundheit waren die Resultate vergleichbar.

In Subgruppenanalysen verstärkte sich der Vorteil der Kombinationsbehandlung. Patienten mit gleichzeitigem Asthma bronchiale (28\% der Gesamtgruppe) hatten eine deutlich niedrigere Wahrscheinlichkeit für Krankenhauseinweisung oder Tod, wenn sie mit der Kombination aus LABA und ICS behandelt wurden (HR 0,84; 95\%-KI 0,77-0,91). Patienten, die keine langwirksamen Anticholinergika erhielten, profitierten besonders (HR 0,79; $95 \%-K I 0,73-0,86$ ).

\section{Fazit}

Die Kombination aus LABA und ICS reduzierte bei Patienten, mit einer neu behandelten COPD, die Mortalität und Krankenhauseinweisungen geringfügig stärker als LABA allein. Patienten, die keine langwirksamen Anticholinergika einnahmen oder an einem Asthma bronchiale litten, profitierten mehr von der Kombinationstherapie, so die Autoren.

\section{Dr. Susanne Krome, Melle}

beitet die pharmazeutische Forschung heute daran, den Wirkstofftransport gezielter zu machen: Wie bei einem Paketdienst soll das Wirkstoffpaket gepackt, gezielt zugestellt und erst vor Ort ausgepackt werden.

Günday-Türeli hat eine neue Methode entwickelt, antibiotische Wirkstoffe in Nano-Behältnissen zu verkapseln. Der Wirkstoff wird dabei nicht verändert und kann hochdosiert geladen werden. Er wird vom Patienten inhaliert, überwindet die Barriere aus Lungenschleim und Biofilm und liefert das Antibiotikum gezielt am Infektionsherd ab. Nach der Wirkstoff-Lieferung werden die NanoTransporter vom Körper abgebaut. Dieses System wurde für Antibiotika entwickelt, es kann aber auch Medikamente gegen verschiedenste Krankheiten zustellen und bspw. auch bei Nahrungsergänzungsmitteln oder Kosmetika zum Einsatz kommen.

Nach einer Mitteilung der Universität des Saarlandes 